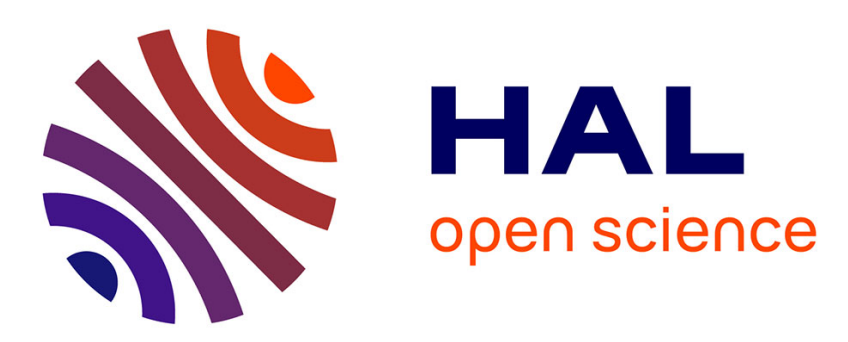

\title{
Combining Synchrony and shape detection to sustain the robot focus of attention on a selected human partner
}

Caroline Grand, Ghiles Mostafaoui, Syed Khursheed Hasnain, Philippe

Gaussier

\section{- To cite this version:}

Caroline Grand, Ghiles Mostafaoui, Syed Khursheed Hasnain, Philippe Gaussier. Combining Synchrony and shape detection to sustain the robot focus of attention on a selected human partner. IEEE Joint conference on Development and learning and on Epigenetic Robotics, Aug 2013, Osaka, Japan. hal-00834548

\author{
HAL Id: hal-00834548 \\ https://hal.science/hal-00834548
}

Submitted on 16 Sep 2013

HAL is a multi-disciplinary open access archive for the deposit and dissemination of scientific research documents, whether they are published or not. The documents may come from teaching and research institutions in France or abroad, or from public or private research centers.
L'archive ouverte pluridisciplinaire HAL, est destinée au dépôt et à la diffusion de documents scientifiques de niveau recherche, publiés ou non, émanant des établissements d'enseignement et de recherche français ou étrangers, des laboratoires publics ou privés. 


\title{
Combining Synchrony and shape detection to sustain the robot focus of attention on a selected human partner
}

\author{
Caroline Grand, Ghiles Mostafaoui, Syed Khursheed Hasnain and Philippe Gaussier \\ Neurocybernetic team, ETIS \\ ENSEA, University of Cergy-Pontoise \\ 95302 France. \\ Emails :caroline.grand at ensea.fr, ghiles.mostafaoui at ensea.fr, syed-khursheed.hasnain at ensea.fr, gaussier at ensea.fr
}

\begin{abstract}
In our previous work we took inspiration from human psychological and neurological data which suggest that synchrony is an important parameter for human-human interaction. We proposed synchrony as a way of interacting and presented a synchrony-based architecture capable of selecting the human partner and of locating the focus of attention. We extend here our approach to people recognition to track the synchronized partner in the context of an autonomous mobile robot. If an agent interacts with a frequency close to the robot's dynamic our architecture selects this agent as an interacting partner and focus on him. Therefor, a shape recognition model learns the shape of the interactant. Combining synchrony and shape strategies, we obtained a bio-inspired robust and efficient architecture to automatically initiate the interaction with a selected partner and to locate and track this preferred partner in real indoor environment using kinematic (synchrony) and form (shape) pathways.
\end{abstract}

\section{INTRODUCTION}

In Human Robot Interaction (HRI), the question of initiating and sustaining the interaction remain difficult to solve. One of the major challenges is to focus the robot visual attention on a human partner. From a biological point of view, in the case of human-human interactions, we know that the human ability to perceive others biological motion is incredibly robust. The exact nature of the visual characteristic permitting us to easily detect biological motion and to focus our attention on it are not clearly defined. Nevertheless, neurobiological and psychological data acknowledged two pathways for biological motion detection : kinematics and shape [1].

For the shape pathway, classical computer vision proposed a tremendous number of published works for people detection, recognition and tracking. One can classify them using different taxonomies (see [2], [3] and [4] for more details). A simple way to differentiate these numerous studies is to consider the use (or not) of explicit models of the human shape. In fact, for people detection and tracking on image sequences, a first possibility is to define explicit $2 \mathrm{D}$ or $3 \mathrm{D}$ models of the human shape to segment and to track (in the successive images) the different body parts of the persons in the visual field (2D silhouette [5], 2D articulated model [6], 3D models [7] etc.). Another possibility is to avoid using a priori explicit knowledge on human shape and to adopt a bottom up approach to construct models for people recognition by combining different low level image characteristics (contours [8], points of interest [9], blobs [10] etc.). In practice, the efficiency of all these promising methods is highly dependent on the application and the experimental conditions (outdoor/indoor, real-time/offline, fixed camera/moving camera etc.).

In the field of bio-inspired Human Robot Interaction the applicability of the previously discussed algorithms is dependent on two main conditions. The first and most obvious one is to maintain a real time interaction between the robot and the human implying the use of algorithms having low cost computational time and not memory resource demanding especially in the case of interactions with mobile robots.

The second condition is more related to biological aspects. In fact, for bio-inspired approaches, the used algorithms must have plausible neural models, moreover, the adopted solution must be, as far as possible, in accordance with neurobiological and psychological data on human visual perception and human-human interaction.

For the kinematic pathway, psychological studies of dyadic interactions and neurobiological data on motor coordination suggested the notion of "synchrony" as an important parameter for early social development as it can be used to initiate and maintain the interaction [11] [12].

In fact, psychological studies on development acknowledged synchrony as a prime requirement for interaction between a mother and her infant. An infant stops interacting with its mother when she stops synchronizing with it [11]. Infants synchronize their legs motion with adult speech [13]. In addition, synchrony detection mechanism in young infants plays a pervasive roll in learning and cognitive development [12] (word learning [14], object interaction skills [15], self-awareness and contorl [16], learning related to self [17] etc.)

An interesting fact is that studies of Interpersonal motor coordination point out unintentional synchronization among people. Issartel et al. studied interpersonal motor-coordination between two participants when they were instructed not to coordinate their movements. The results showed that participants could not avoid unintentional coordination with each other [18]. This reflects that when visual information is shared 
between two people in an interpersonal situation, they coordinate (unintentionally) with each other.

Keeping in view the importance of synchrony in social interaction, it has also been widely studied and used in robotics. Andry et al. proposed synchrony as an internal reward for learning [19]. Prepin and al. also used the level of synchrony as a reinforcement signal for learning [20]. Blanchard and Canamero proposed a velocity detection system to synchronize the movements of two robots to improve the reactivity of agents to changes in their environment [21]. Marin et al. underlined that motor resonance between robots (humanoid) and humans could optimize the social competence of human-robot interactions [22]. Michalowski et al. developed a dancing robot to analyses the properties and significance of synchronized movement in general social interaction [23].

In the line of this state of the art, we recently proposed a synchrony-based neural network architecture capable of selecting the robot partner and of locating its focus of attention in order first to initiate and then to sustain the interaction [24]. This modal was validated by experimental and psychological studies [25].

Using this previous architecture for initiating the humanRobot interaction, we will question in this paper the emergence, from a learning stage during interactions, of a knowledge about a pattern of the human partner appearance (shape). We will also study the focus of attention using two different visual modalities : motion (optical flow) for a synchrony based focus of attention and pattern recognition for a shape detection based focus of attention.

More practically, we are aiming to give to a Robulab mobile robot equipped with a camera the ability to :

- initiate automatically an interaction on a selected human partner in the basis of synchrony detection

- learn automatically during interactive games its partner shape

- focus its attention and track the human partner using both synchrony detection and shape recognition

To tackle these harsh questions, we will detail in this paper a bio-inspired and developmental approach inspired by psychological and neurobiological studies.

\section{EXPERIMANTAL SETUP.}

Our experimental setup is composed by a Robosoft robulab 10 equipped with four wheels, two for directions and two for stabilization, a proximity sensors for obstacle avoidance, an embedded computer, and for the visual perception, a pantilt camera controlled with a SSC-32 card through a serial communication. Only the "pan" rotation of the camera is used. The experiments were performed in an indoor environment (See Figure 1).

\section{SYNCHRONY BASED ATTENTIONAL MECHANISM AND PARTNER SELECTION}

As stated above, we will use a model developed in [24] for initiating the interaction and selecting the partner on the

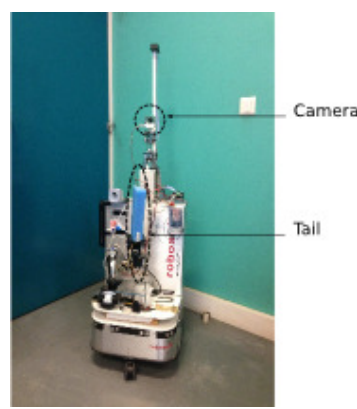

Fig. 1. Experimental Setup

basis of synchrony detection. A summarized definition of this model is given in this section.

As illustrated Figure 2, the model can be divided into three complementary architectures. The first (part A) is related to a simple model for dynamical interaction. The second one (part $\mathrm{B}$ ) is dedicated to partner selection and the last one completes the model by adding an attentional mechanism for locating the partner.

As a first step towards human-robot interactions, we use a simple dynamical interaction model (figure 2, part a) to provides minimal abilities to the robot to interact with humans in the basis of synchrony by adopting the phase and frequency of its partner. Figure 2(A) (dotted box) shows the oscillator module [26] controlling the robot motion dynamics (oscillations). It consists of two neurons $N 1$ and $N 2$ inhibiting each other proportionally to the variable $\beta$. The oscillating frequency is a function of the variables $\alpha 1, \alpha 2$ and $\beta$ :

$$
\begin{aligned}
& N_{1}(n+1)=N_{1}(n)-\beta N_{2}(n)+\alpha 1 \\
& N_{2}(n+1)=N_{1}(n)+\beta N_{2}(n)+\alpha 2
\end{aligned}
$$

Normally, the robot's oscillator, representing the internal dynamic of the robot, oscillates at its own frequency and amplitude. If a human interacts with the agent by moving its arm, the motion in the visual field is estimated by an optical flow algorithm, the velocity vectors are then converted into positive and negative activities. If the perceived movements are in the upward direction, the oscillator gets the positive activity and its amplitude increases on the positive side. On the contrary, if the negative activity is perceived, the amplitude goes down. Lets now rephrase the mathematical equation of the robot oscillator by :

$$
N_{1}(n+1)=N_{1}(n)-\beta N_{2}(n)+\alpha 1+f^{\prime}
$$

Where $f^{\prime}$ is the energy induced by the optical flow activities. Consequently, when an agent interacts, depending on the visual energy (optical flow) produced by its movements, the robot's oscillator will be modified (frequency and phase) within certain limits defined by a coupling factor to avoid saturations .

This first model provides a basic architecture initiating automatically a human-robot interaction by synchronizing the agent's movements (in an imitating framework). The Part (b) of Figure 2 describe a selection of partner model completing the previous one to be capable of choosing an interacting partner among various interacting agents. It can be segregated into 


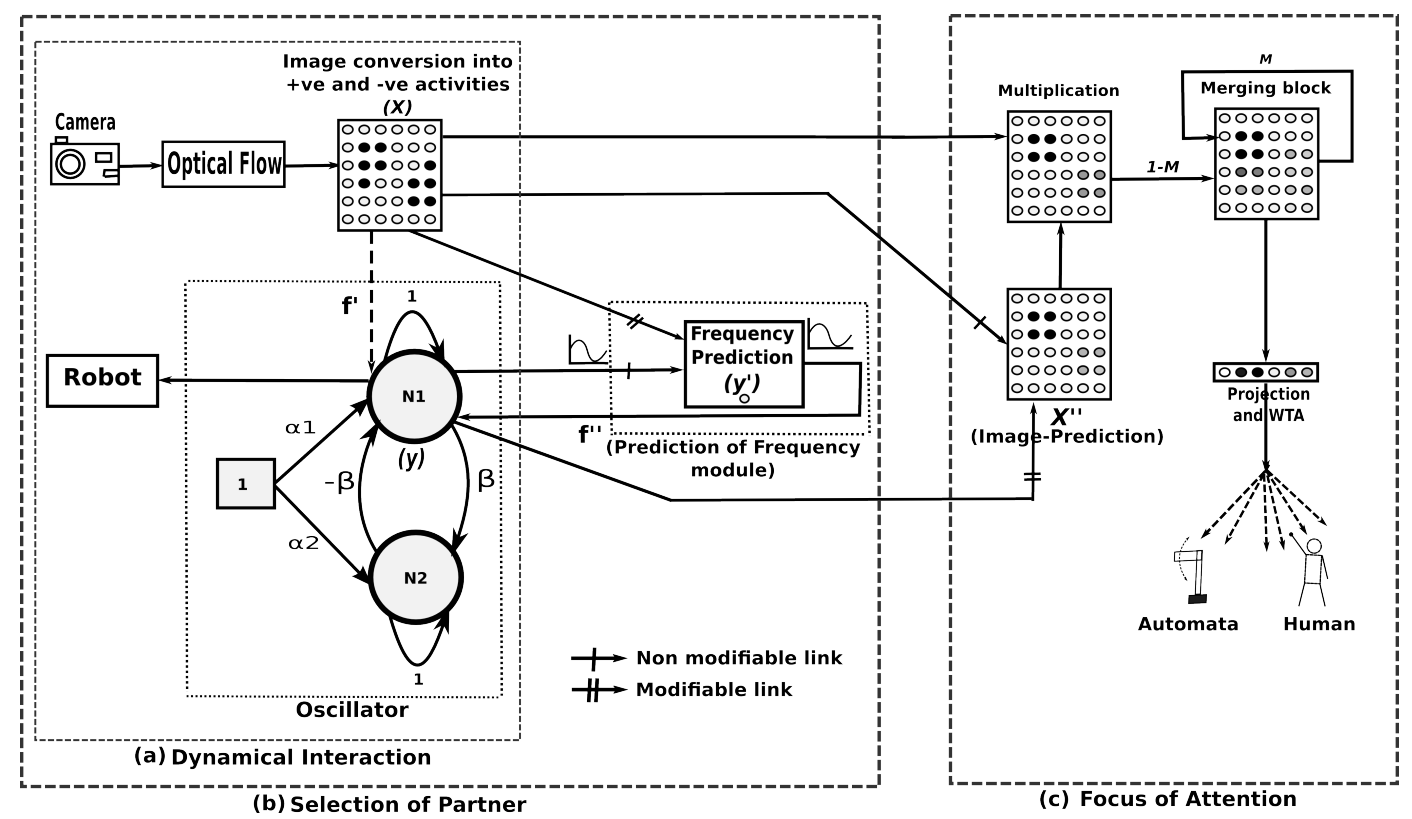

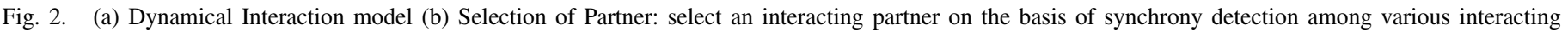
agents. (c) Focus of Attentional: locate and point out the synchronized interacting agent defined by the selection of partner algorithm.

two parts. The first one is the dynamical interaction model (part a) and the other one is the frequency-prediction module. Previously, the robot's oscillator was directly linked to the external visual stimuli $f^{\prime}$ now, the coupling activities are linked with the frequency-prediction module (f'). The equation 3 can be rephrase as

$$
N_{1}(n+1)=N_{1}(n)-\beta N_{2}(n)+\alpha 1+f^{\prime \prime}
$$

Where, $f^{\prime \prime}$ is the coupling energy feed by the frequencyprediction block. The other variables remain unchanged.

The selection of partner architecture works in two phases: a learning phase and a testing phase. During the learning phase, the robot perceive and learns its own dynamics. It initiates two processes. First the frequency-prediction module starts now predicting the robot's modifiable oscillator as a weighted sum of its own visual stimuli. As a consequence, it also modifies the robot's oscillator. This process of modifying, learning and adapting continues and converge after some time.

After this learning, when an agent interacts with a frequency close to the learnt one, weights (that are already learnt on modifiable links) are associated with the visual activities induced by the human movements and the robot's modifiable oscillator adopts the interactant frequency and phase. If the interacting frequency is different from the learnt one, the weights could not be associated with the visual stimuli and the robot continues to move at its default frequency. The same is true in the case of multiple interacting agents. Among several interactants, only the agent having a similar dynamic (frequency) as the robot is selected.

However, using the selection of partner, the robot will not be able to locate the good interacting partner in its visual field, because this algorithm works on the perceived energy irrespective of the spatial information (agent location).
Figure 2(c) shows the Focus of attention (FOA) architecture. Here, if an agent interacts with a frequency close to the robot dynamics, the image-prediction block $\left(X^{\prime \prime}\right)$ learns the locations of the interactant movements. To discriminate between multiple stimuli, our algorithm modulates the current visual stimuli with the image-prediction $X^{\prime \prime}$. A merging block is used to calculate a weighted average of these current results (modulation) and the results of the previous iteration. The higher values of this merging block are then correlated to the location of synchronous movements. All the pixels of the merging block are projected on the $x$ axis. A Winner Takes All (WTA) selects the highest activated column. This selected column indicates the location of the synchronized movement.

We use a Least Mean Square (LMS) [27] algorithm for the learning of the image-prediction $\left(X^{\prime \prime}\right)$ and the frequencyprediction module.

\section{LEARNING AND RECOGNITION OF THE PARTNER SHAPE}

For the learning and recognition of the shape partner, it is clear that we can not consider the use of explicit 2D or 3D models as we are aiming for a developmental approach. Additionally, using explicit complex 2D or 3D models imply an increased computational complexity which is not allowable for real time applications on mobile robots. Consequently, our approach consists on using a bottom up algorithm permitting to learn the partner's shape (while interacting) using points of interest as low level visual features. The motivation behind the choice of points of interest for the shape characterization is (besides the reasonable computational time) the direct similitude with the human eye saccadic movements.

\section{A. A general model for object recognition}

We define, as a first step to recognize the partner shape, a general model for object recognition inspired by the works 
in [28] and [29]. The general principal of this model is to learn local views of the objects in the basis of point of interest detection. As illustrated Fig. 4, the spatial gradient information is first extracted from the grayscale images. The resulted image gradient is then convolved by a DOG (Difference Of Gaussian) filter. The output of this process is a saliency map which highlight regions in the image having a local structure in the form of corners. Local maxima are then selected from this saliency map.

Local views collecting the pixel around each detected interest points (here with a radius of 20 pixels) are then extracted and filtered by a log polar transform in order to be robust to scale changing and rotational variations. The filtered local views feed the Selective Adaptive Winner (SAW) which is an ART-based neural networks. Depending on the vigilance threshold of the SAW, if the new inputs (local views) are too different from the previous ones, new encoding neurons are recruited. A Winer Take All (WTA) is then used to select the winning local views.

The model presented in (Fig. 4) can after be divided into two parts. the recognition of what is the object, and the localization of "where" is the object. A first LMS (Least Mean Square) algorithm is used for the "what" pathway to learn the local views associated to each object. The number of neurons in the LMS is then corresponding to the possible number of objects to learn. Regarding to the Where pathway, two LMS are used to associate the object center position respectively on the $\mathrm{x}$ and $\mathrm{y}$ axis relative to the local views belonging to it ("what" pathway). As presented figure 3, after the learning phase, each selected local view (point of interest) will have its own prediction of the object center. If most of them predict the same position, the object will be well recognized. In the opposite case, several positions of the object center will be predicted without a majority vote permitting to identify winner (see figure $3 \mathrm{D}$ ). If an object is learned at a given position and detected in another one, the output of the LMS shift the learn position relative to the actual position allowing to predict the object position.
A.

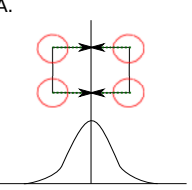

B.

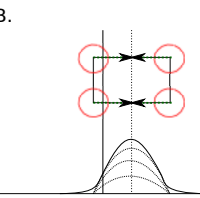

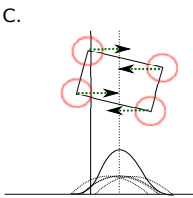

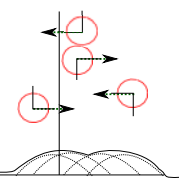

Fig. 3. Object recognition. A) Learning of the object position in the center of the image. B) After learning : prediction of shifted object position. C) Influence of rotation on prediction. D) Detection of interest points which predict different positions of the object center

\section{B. Attentional Mechanism and human partner shape learning}

The first model for selecting a partner (section III) locate the robot's focus of attention on regions of interest by detecting synchrony between the robots internal dynamics and the interactant's movements, therefor, the robots focus of attention can not be maintained if the human stops moving. For realistic human robot interactions, the agents must be able to switch their roles (turn taking) leading to different phases of interactions where one of the interactants (or both) can eventually stop moving. In this case, to sustain the robot's focus of attention on the correct partner we developed a new attentional mechanism gathering the two previous models to locate the robots visual focus on its partner by both synchrony and shape detection.

For learning the human partner shape, we will use the model detailed section IV-A for object recognition. To ensure that the robot will learn automatically the shape of the correct human partner (rather than other humans or objects), we added the following conditions :

- the shape learning starts only after the robot focuses its attention on the selected partner by synchrony detection to ensure the presence in the visual field of the correct human interactant

- the saliency map resulting from the filtering by the DOG is modulated par the motion intensity (optical flow) in order to focus most of the selected local views on the moving human partner (while interacting with the robot).

This model works as follow : first, the robot must learn a preferred frequency of interaction by perceiving a human partner moving his arm with a certain dynamic. After the learning stage, if a human start moving with a dynamic close the learnt frequency of interaction, the robot will be able to select and locate the human partner in the visual field in the basis of synchrony detection by using the model explained in section III.

The predicted position of the human partner is then used to move the robot toward the partner using a Neural Field [30] which allow a stable motor control. The neural field population activity is described by the following differential equation :

$\tau \frac{d(u(\phi, t))}{\delta t}=-u(\phi, t)+S(\phi, t)+h+\int_{Z} w(\phi-z) f\left(u\left(\phi^{\prime}, t\right)\right) \mathrm{d} z$

$\tau$ is the temporal integration parameter, $S(\phi, t)$ is the input of the neuron $\phi$ at a given time $t$ and $u(\phi, t)$ the neurons output activity. The last term correspond to a convolution with an integration kernel. Here, the temporal integration parameter allow remaining activities in neurons corresponding to the previous locations where the partner was recognized. The size of the integration kernel corresponds to a competition parameter.

Hence the synchrony based focus of attention moved the robot toward the human interacting partner the shape recognition model start to learn the human shape by selecting salient local views in regions with high motion intensity (optical flow). Statistically, after a while, most of the selected local views will belong to the human partner.

To maintain a good recognition of the human, the robot must learn different views of its partner. To obtain this refinement of the partner shape model we introduced a vigilance signal. If the recognition rate of the learnt local views is very low the partner is not detected. For a higher rate (more than $60 \%$ ), the human is detected and his shape model remain unchanged. If the rate is between 10 and $60 \%$, the human is detected but new local views are selected and added to the shape model. 


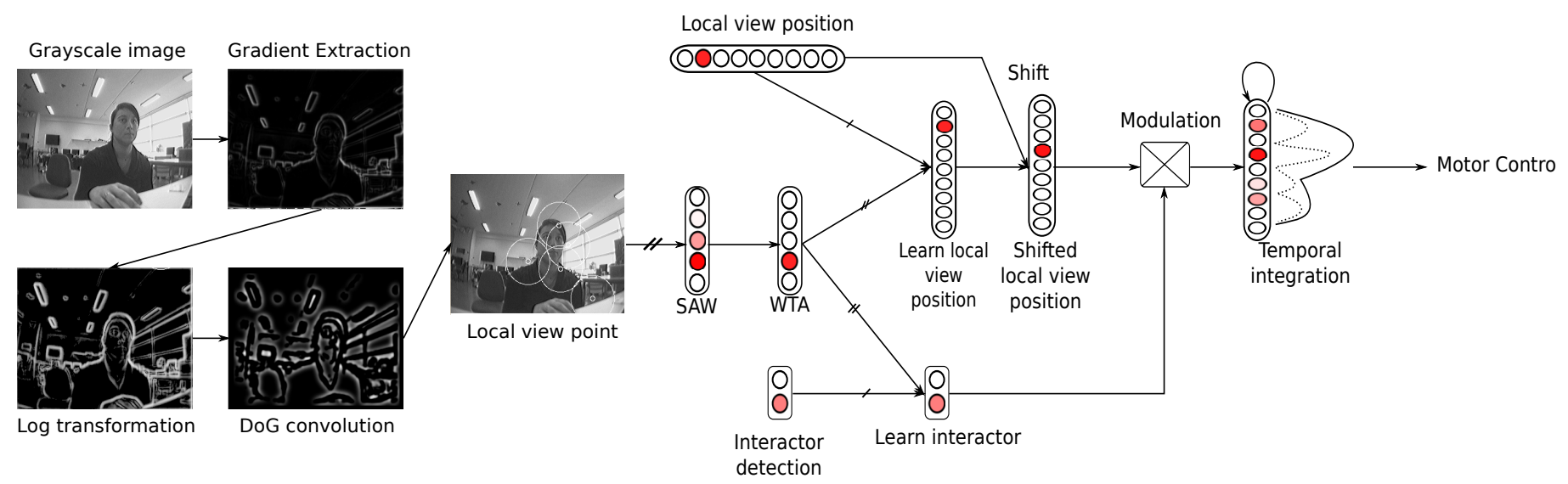

Fig. 4. General model for object recognition

Therefor, we obtain two different strategies to locate and track the human partner by focusing the robot attention on it. The first is based on the motion information and synchrony detection and the second one on shape recognition. Each strategy has its own auto-evaluation, the vigilance signal for the shape recognition and the coupling factor permitting the synchrony detection for the other strategy.

By feeding the neural field by the sum of the neurons encoding the partner location in the two strategies as in figure 5 , we obtain a system capable of locating the partner using only synchrony detection or shape recognition or a combination of the two strategies.

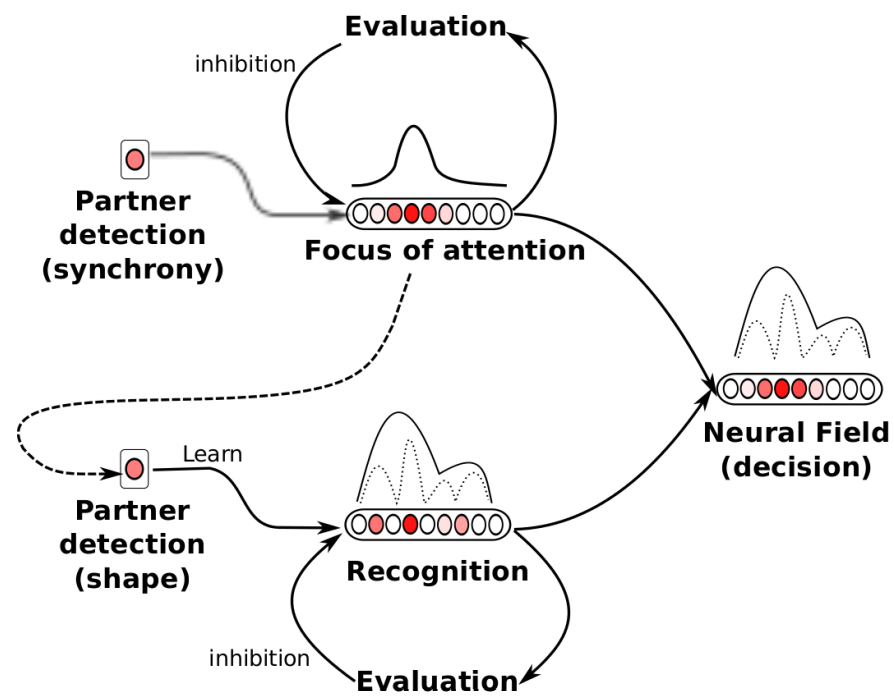

Fig. 5. Combining synchrony detection and shape recognition to locate the partner

\section{EXPERIMENTAL RESULTS.}

In order to test the model capacity to detect the partner, we have done experimental tests in real non constrained indoor environment with the Robulab robot. First a human partner move his arm in front of the robot to make it learn a preferred frequency of interaction. After this learning phase, when a human interact with the robot with a dynamic close to the learnt one (the learned frequency can be seen thanks to the oscillatory movements of the tail embedded on the robot, see figure 1), the robot select and locate this preferred partner in the basis of synchrony detection. Consequently, it starts to learn the shape of the partner.

At the end of these learning phases, the robot is able to locate and focus its attention on the human partner by combining synchrony detection and shape recognition. The robot is then capable of tracking its partner. in (fig. 6) we can see a comparison between a ground truth of the position of the human in the visual field and the positions predicted by our model demonstrating the efficiency of this combined strategy. To validate the interest points (or the local views) selection, we also illustrated in this figure the positions of all the local views including the ones belonging to the partner (black plain point) and the others.

We can notice the presence of a prediction latency of 2 or 3 seconds. We explain that by the presence of temporal integrations for partner shape recognition.

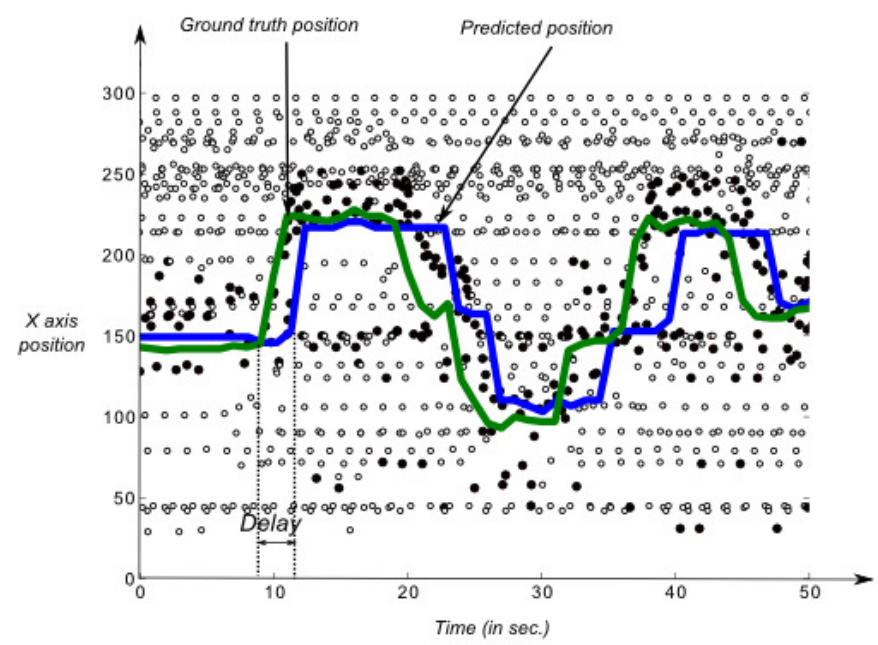

Fig. 6. Evolution of the ground truth (green) and the predicted (blue) position of the partner

We also tested the robustness of our shape recognition after the learning step by adding an unknown human in the visual 
field. It is worth noticing that if this second human interact with a frequency which differ from the learnt one, the robot will keep its focus of attention on the preferred partner. In the contrary (or if the two human stops moving) the robot must discriminate these two stimuli using shape recognition.

This case is illustrated in Fig. 7 where the preferred partner is on the right side while the human distractor is on left side. A statistical analysis showed that $58 \%$ of the points of interest (red points) belonging to the preferred partner have been well classified, $42 \%$ have been wrongly predicted, $15 \%$ on the distractor and $27 \%$ are noise from the background (white points). This analysis show that the robot is able to recognize the preferred partner even in the presence of other possible human interactant.

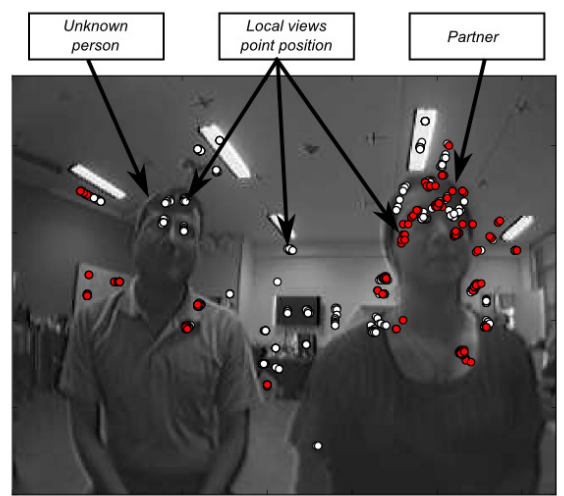

Fig. 7. Image from the experiments : on the right side we can see the learned partner and on the left the distractor. The dot points represents the selected points of interest. In red, the one recognized as the belonging to the partner, in white the ones which are classified as noise.

Lets now consider the complete scenario as illustrated in figure 8 . First the mobile robot start moving by focusing its attention on random regions of the visual field because of the lack of salient regions of interest. At time $t=40$ seconds, a human start interacting with a frequency close to the one learned by the robot. Consequently, the synchrony based focus of attention select and predict the location of the human partner (blue line in figure 8). The neural Field controlling the robots movements turn the Robulab toward the partner and center him in the image (black line in figure 8). When the robot attention is focused on the partner in the basis of synchrony detection, the shape learning is activated (green areas in figure 8 ). From time $t=40$ to $t=80$, we can notice that the shape learning is stopped (red areas in figure 8) and reengaged relative to the establishment or not of the synchrony based focus of attention. A refined learning of the partner shape is consequently obtained. As we can see figure 8, starting from $t=80$, even if the robot lost the synchrony, if the human move to the left side or the right side, the robot track its partner and move toward his direction in the visual field using shape recognition.

\section{CONCLUSION AND PERSPECTIVES}

In this paper, we presented a new approach complementing our previous model for select an interacting partner among multiple agents based on synchrony detection. Once the partner is selected, our architecture learns the shape of interacting

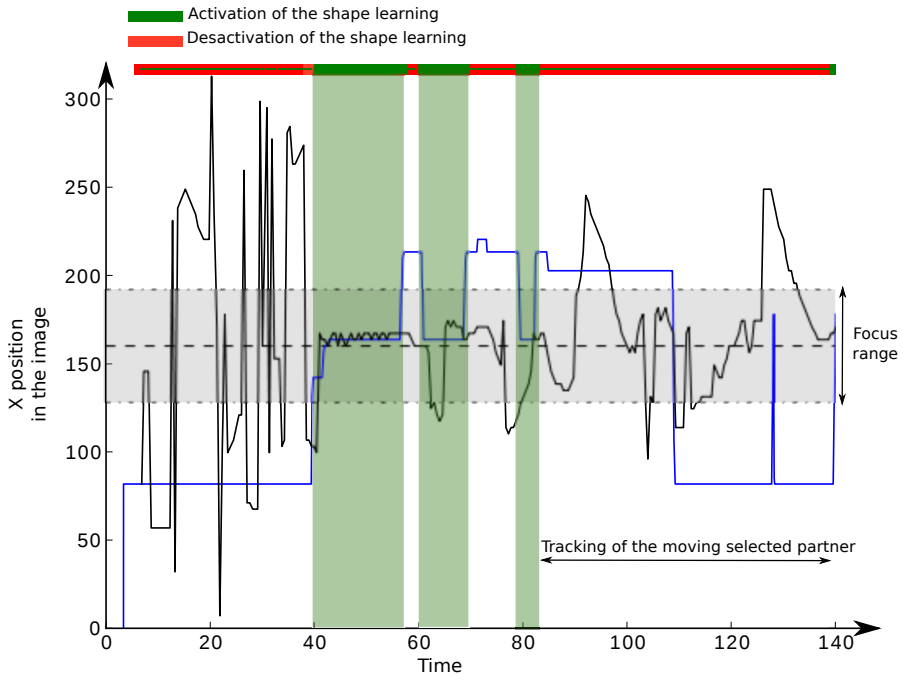

Fig. 8. Partner detection and tracking : Experimental results in real conditions by combining synchrony based and shape based strategies

partner, the robot is then capable of locating and following the recognized agent in the basis of shape recognition. The results of our previous work already demonstrated that if several agents are trying to interact (with a robot), the robot selects the partner which has the similar frequency of interaction as the robot has. Moreover, focus of attention algorithm forces the robot to turn the robot in the direction of the synchronized agent. However, if the selected agent stops synchronizing, it may not be located. Here, we defined a model adding a shape recognition model which learn the partner shape in a developmental manner after initiating the interaction in the basis of synchrony. By combining these two strategies (synchrony and shape) we gave to the robot new capabilities to focus on and track the correct partner even when the interaction is stopped permitting the emergence of possibilities to re-engage the interaction (using synchrony or shape). As a future work, we are planing to use synchrony detection, focus of attention and shape recognition in turn-taking games and joint attention strategies on a mobile robot which will initiate interactions by synchronizing first with the leg movements frequency of a human possible partner.

\section{ACKNOWLEDGMENT}

This work was supported by the "Interact" French project referenced ANR-09-CORD, the "Neurorobot" French ANR project and the DGR (Direction General de l'Armement)

\section{REFERENCES}

[1] Joachim Lange and Markus Lappe, The role of spatial and temporal information in biological motion perception, Advances in Cognitive Psychology, 2007 volume 3 no 4 419-429

[2] J.K. Aggarwal and Q. Cai. Human motion analysis: a review. Computer Vision and Image Understanding, 73(3):428440, 1999.

[3] D.M. Gavrila. The Visual Analysis of Human Movement: A Survey. Computer Vision and Image Understanding, 73(1), January 1999.

[4] T.B. Moeslund and E. Granum. A Survey of Computer Vision-Based Human Motion Capture. Computer Vision and Image Understanding, 81(3), March 2001. 
[5] L.S. Davis, D. Harwood, and I. Haritaoglu. Ghost: A human body part labeling system using silhouettes. ICPR, 1998.

[6] T.J. Cham and J.M. Rehg. A multiple hypothesis approach to figure tracking. CVPR, pages 239245, 1999

[7] J. Rehg and T. Kanade. Visual tracking of high dof articulated structures : an application to human hand tracking. Proc. of European Conference on Computer Vision, pages 3546, 1994.

[8] R. Goldenberg, R. Kimmel, E. Rivlin, and M. Rudzsky. Fast g?eodesic active contours. Scale Space, pages 3445, 2002.

[9] P. Gabriel, J.B. Hayet, J. Piater, and J. Verly. Object tracking using color interest points. IEEE International Conference on Advanced Video and Signal based Surveillance, pages 159164, 2005.

[10] R. Fablet and M.J. Black. Automatic detection and tracking of human motion with a view-based representation. ECCV, page I $18 \mathrm{ff}, 2002$.

[11] J. Nadel, I. Carchon, C. Kervella, D. Marcelli, D, Reserbat. Expectancies for social contingency in 2 months old. Developmental science 2 (1999) 164-173.

[12] In Berthouze, L., Kozima, H., Prince, C. G., Sandini, G., Stojanov, G., Metta, G., and Balkenius, C. (Eds.). C. G. Prince, G. J. Hollich, N. A Helder, E. J. Mislivec, A. Reddy, S. Salunke and N. Memon. Proceedings of the Fourth International Workshop on Epigenetic Robotics. Lund University Cognitive Studies, 117, ISBN 91-974741-3-4

[13] W.S.Condon and L. W. Sander (1974). Neonate movement is synchronized with adult speech: Interactional participation and language acquisition. Science, 183, 99101.

[14] L. J. Gogate and L. E. Bahrick (1998). Intersensory redundancy facilitates learning of arbitrary relations between vowel sounds and objects in seven-month-old infants. Journal of Experimental Child Psychology, 69, 133-149.

[15] J. S. Watson (1972). Smiling, cooing, and The Game. Merrill-Palmer Quarterly, 18, 323-339.

[16] G. Gergely and J. S. Watson (1999). Early socio-emotional development: Contingency perception and the social-biofeedback model. In: P. Rochat (Ed.), Early Social Cognition: Understanding Others in the First Months of Life (pp. 101-136). Mahwah, NJ: Lawrence Erlbaum.

[17] P. Rochat and T. Striano (2000). Perceived self in infancy. Infant Behavior \& Development, 23, 513-530.

[18] J. Issartel, L. Marin, M. Cadopi. Unintended Interpersonal Coordination: Can we march to the beat of our own drum?. Neuroscience Letters, 411:174-179, 2007.

[19] P. Andry, A. Blandchard, P. Gaussier. Using the rhythm of non-verbal human-robot interaction as a signal for learning. IEEE Transactions on Autonomous Mental Development 3(1): 30-42 (2011).

[20] K. Prepin and P. Gaussier. How an agent can detect and use Synchrony parameter of its own interaction with a human. COST 2102 Training School 2009: 50-65.

[21] A. Blanchard and L. Caamero. Using visual velocity detection to achieve synchronization. In Y. Demiris (Ed.), AISB05 third International Symposium on imitation in Animals and Artifacts. Hatfield UK. 2005.

[22] L. Marin, J. Issartel and T. Chaminade. Interpersonal motor coordination, from humanhuman to humanrobot interactions.In: Dautenhahn, Kerstin (ed.), Robots in the Wild: Exploring human-robot interaction in naturalistic environments: Special Issue of Interaction Studies 10:3 (2009).

[23] M. P. Michalowski, S. Sabanovic and H. Kozima. A dancing robot for rhythmic social interaction. Proceedings of the ACM/IEEE international conference on Human-robot interaction ACM New York, NY, USA 2007

[24] S.K.Hasnain, P. Gaussier, G.Mostafaoui. Synchrony as a tool to establish focus of attention for autonomous robots, IEEE Intelligent Robots and Systems (IROS 2012), Pages: 2423- 2428

[25] S.K.Hasnain, G.Mostafaoui, R.Salesse, L.Marin and P. Gaussier, Intuitive HR Interaction based on unintentional synchrony: a psychoexperimental study, Submitted to ICDL-EPIROB 2013

[26] A. Revel, P. Andry : Emergence of Sturctured Interactions: From theoretical model to pragmatic robotics. Neural Networks, vol. 22, no. 2, pp. 116-125 - 2009.

[27] B. Widrow, M. E. Hoff. Adaptive switiching circuits. In IRE WESCON, pages 96-104, New York, 1960. Convention Record.
[28] Gaussier, P. and Joulain, C. and Zrehen, S. and Banquet, JP and Revel, A, Visual navigation in an open environment without map, Proceedings of the 1997 IEEE/RSJ International Conference on Intelligent Robots and Systems, 1997, volume 2, pages 545-550

[29] Lepretre, Sacha and Gaussier, Philippe and Cocquerez, Jean-pierre. From navigation to active object recognition. Citeseer. 2000

[30] S.Amari. Dynamics of pattern formation in lateral-inhibition type neural fields (1977). Biological cybernetics, 27(2), pages 77-87. 